\title{
Developing 1D MM of Axisymmetric Transient Quenched Chromium Steel to Determine LHP
}

\author{
Abdlmanam S. A. Elmaryami and Badrul Omar \\ Mechanical Engineering Department, Universiti Tun Hussein Onn Malaysia, Batu Pahat, Johor, 86400 Parit Raja, Malaysia \\ Correspondence should be addressed to Abdlmanam S. A. Elmaryami, damer604@yahoo.com
}

Received 4 November 2011; Revised 1 January 2012; Accepted 8 January 2012

Academic Editor: Elena V. Pereloma

Copyright ( 2012 A. S. A. Elmaryami and B. Omar. This is an open access article distributed under the Creative Commons Attribution License, which permits unrestricted use, distribution, and reproduction in any medium, provided the original work is properly cited.

\begin{abstract}
The modelling of an axisymmetric industrial quenched chromium steel bar AISI-SAE $8650 \mathrm{H}$ based on finite element method has been produced to investigate the impact of process history on metallurgical and material properties. Mathematical modelling of 1-dimensional line (radius) element axisymmetric model has been adopted to predict temperature history and consequently the hardness of the quenched steel bar at any point (node). The lowest hardness point (LHP) is determined. In this paper hardness in specimen points was calculated by the conversion of calculated characteristic cooling time for phase transformation $t_{8 / 5}$ to hardness. The model can be employed as a guideline to design cooling approach to achieve desired microstructure and mechanical properties such as hardness. The developed mathematical model is converted to a computer program. This program can be used independently or incorporated into a temperature history calculator to continuously calculate and display temperature history of the industrial quenched steel bar and thereby calculate LHP. The developed program from the mathematical model has been verified and validated by comparing its hardness results with commercial finite element software results.
\end{abstract}

\section{Introduction}

Quenching is a heat treatment usually employed in industrial processes in order to control mechanical properties of steels such as hardness [1]. The process consists of raising the steel temperature above a certain critical value, holding it at that temperature for a specified time and then rapidly cooling it in a suitable medium to room temperature [2]. The resulting microstructures formed from quenching (ferrite, cementite, pearlite, upper bainite, lower bainite, and martensite) depend on cooling rate and on chemical composition of the steel [3].

Quenching of steels is a multiphysics process involving a complicated pattern of couplings among heat transfer, because of the complexity, coupled (thermal-mechanicalmetallurgical) theory, and nonlinear nature of the problem, no analytical solution exists; however, numerical solution is possible by finite difference method, finite volume method, and the most popular one-finite element method (FEM) [4]. During the quenching process of the steel bar, the heat transfer is in an unsteady state as there is a variation of temperature with time [5]. The heat transfer analysis in this paper will be carried out in 3-dimensions. The three dimensional analysis will be reduced into a 1-dimensional axisymmetric analysis to save cost and computer time $[4,6$, 16]. This is achievable because in axisymmetric conditions, there is no temperature variation in the theta $(\boldsymbol{\theta})$ direction and in $(\mathbf{z})$ direction, the temperature deviations are only in (r). The Galerkin weighted residual technique is used to derive the mathematical model. In this paper, 1-dimensional line (radius) element will be developed.

\section{Mathematical Model}

The temperature history of the quenched cylindrical steel bar at any point would be calculated; three-dimensional heat transfer can be analyzed using one dimensional axisymmetric elements as shown in Figures 1, 2, and 3.

The linear temperature distribution for an element (radius) line, $T$ is given by:

$$
T^{(R)}=a_{1}+a_{2} R,
$$




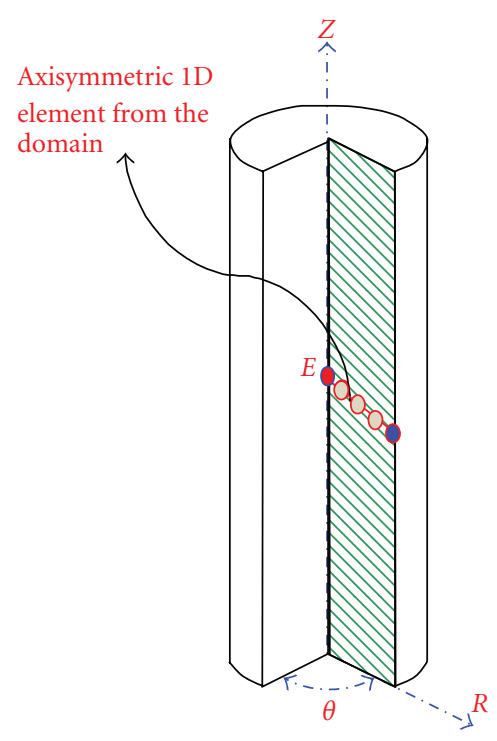

FIGURE 1: The axisymmetric one-dimensional line (radius) element from the domain, on the cylindrical chromium steel bar which had been heated and then submerged in sea water.

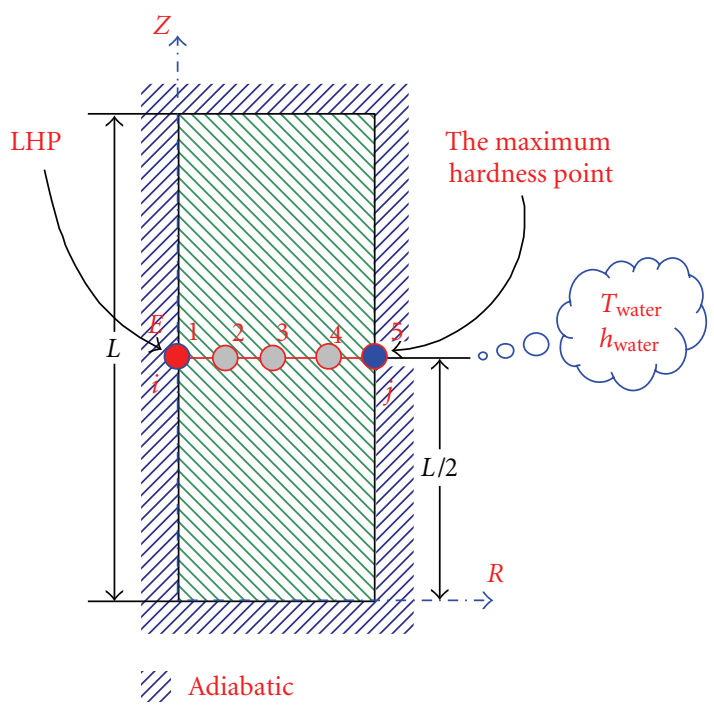

Figure 2: The axisymmetric one-dimensional line (radius) element from the domain on the axisymmetric rectangular cross section.

where $T(R)=$ nodal temperature as the function of $R, a_{1}$ and $a_{2}$ are constants, $R$ is any point on the (radius) line element.

2.1. Shape Function of the Axisymmetric Triangular Element. The shape functions were to represent the variation of the field variable over the element. The shape function of axisymmetric 1-dimensional line (radius) element expressed in terms of the $r$ coordinate and its coordinate as shown

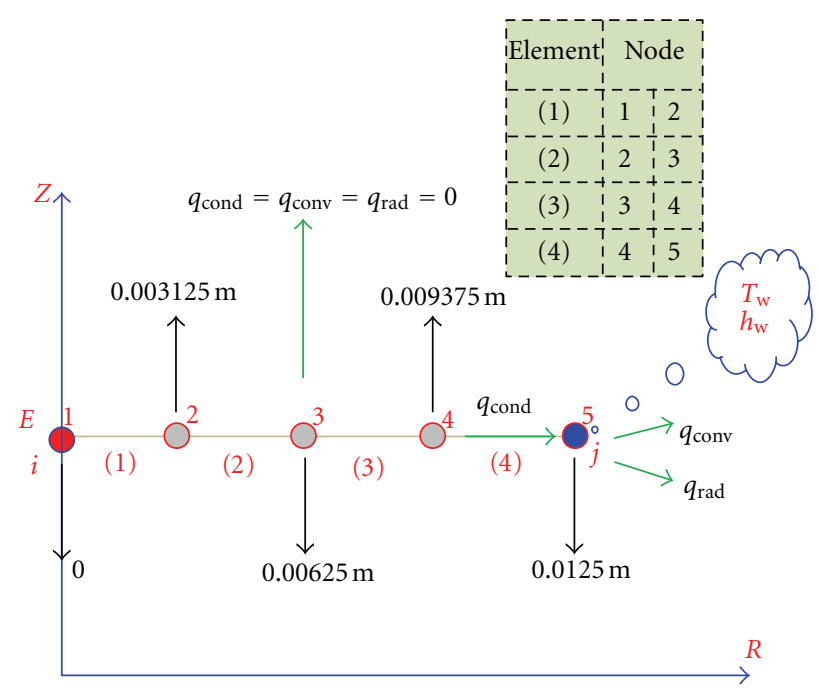

FIGURE 3: The axisymmetric one dimensional line (radius) element from the domain, the selected 4 elements with 5 nodes and the boundary at node $j(5)$ for an element 4 .

in Figure 4; which are derived to obtain the following shape functions as shown:

$$
\begin{gathered}
S_{i}=\left(\frac{R_{j}-R}{R_{j}-R_{i}}\right)=\left(\frac{R_{j}-R}{L}\right), \\
S_{j}=\left(\frac{R-R_{i}}{R_{j}-R_{i}}\right)=\left(\frac{R-R_{i}}{L}\right) .
\end{gathered}
$$

Thus the temperature distribution of $1 \mathrm{D}$ radius for an element in terms of the shape function can be written as:

$$
T^{(R)}=S_{i} T_{i}+S_{j} T_{j}=\left[S^{(r)}\right]\{T\},
$$

where $\left[\mathrm{S}^{(r)}\right]=\left[S_{i} S_{j}\right]$ is a row vector matrix and

$$
\{T\}=\left\{\begin{array}{c}
T_{i} \\
T_{j}
\end{array}\right\}
$$

is a column vector of nodal temperature of the element.

Equation (3) can also be expressed in matrix form as:

$$
T(R)=\left[\begin{array}{ll}
S_{i} & S_{j}
\end{array}\right]\left\{\begin{array}{c}
T_{i} \\
T_{j}
\end{array}\right\} .
$$

Thus for 1-dimensional element we can write in general:

$$
\Psi^{(e)}=\left[\begin{array}{ll}
S_{i} & S_{j}
\end{array}\right]\left\{\begin{array}{l}
\psi_{i} \\
\psi_{j}
\end{array}\right\},
$$

where $\Psi_{i}$ and $\Psi_{j}$ represent the nodal values of the unknown variable such as in our case temperature also the unknown can be deflection, velocity, and so forth.

2.2. Natural Area Coordinate. Using the natural length coordinates and their relationship with the shape function by simplification of the integral of Galerkin solution. 


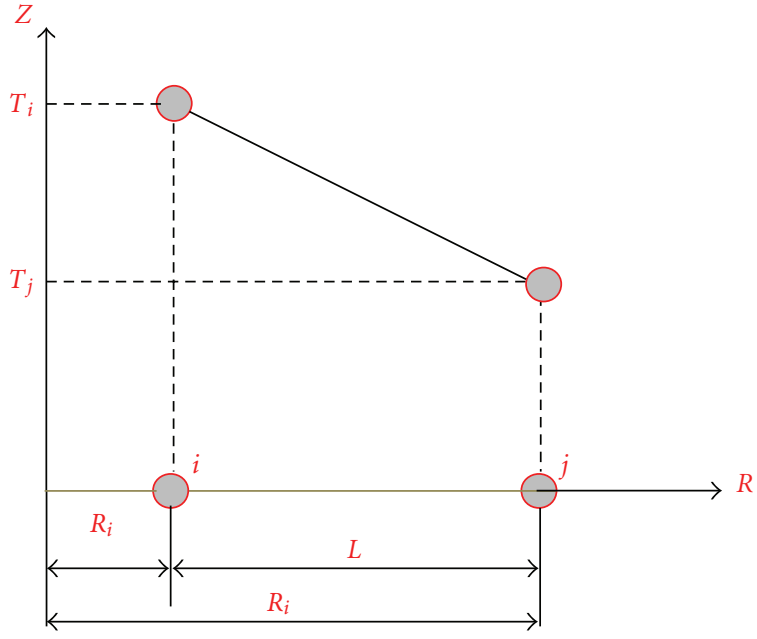

FIGURE 4: One-dimensional linear temperature distribution for an element (radius) line in global coordinate system.

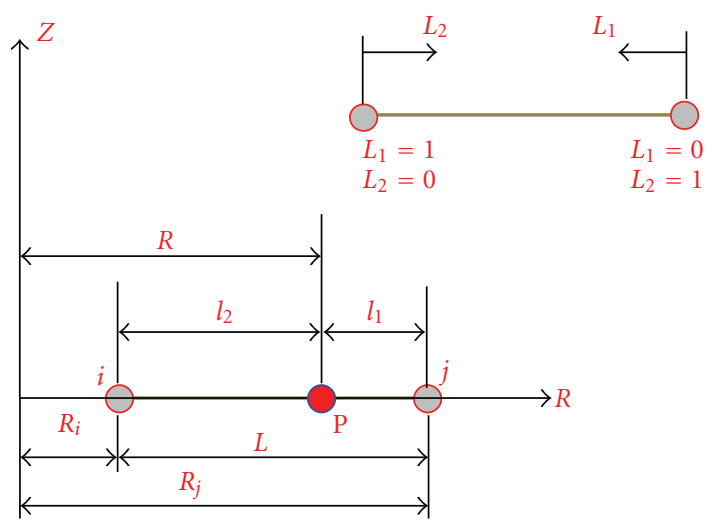

FIgURE 5: Two-node line element showing interior point $\mathrm{P}$ and the two naturals coordinates $L_{1}$ and $L_{2}$.

The two length natural coordinates $L_{1}$ and $L_{2}$ at any point $\mathrm{P}$ inside the element are shown in Figure 5 from which we can write:

$$
\begin{aligned}
& L_{1}=\frac{R_{j}-R}{R_{j}-R_{i}}=\frac{l_{1}}{L}, \\
& L_{2}=\frac{R-R_{i}}{R_{j}-R_{i}}=\frac{l_{2}}{L} .
\end{aligned}
$$

Since it is a one-dimensional element, there should be only one independent coordinate to define any point $\mathrm{P}$. This is true even with natural coordinates as the two natural coordinates $L_{1}$ and $L_{2}$ are not independent, but are related as:

$$
L_{1}+L_{2}=1 \quad \text { or } \quad L_{1}+L_{2}=\frac{l_{1}}{L}+\frac{l_{2}}{L}=1 .
$$

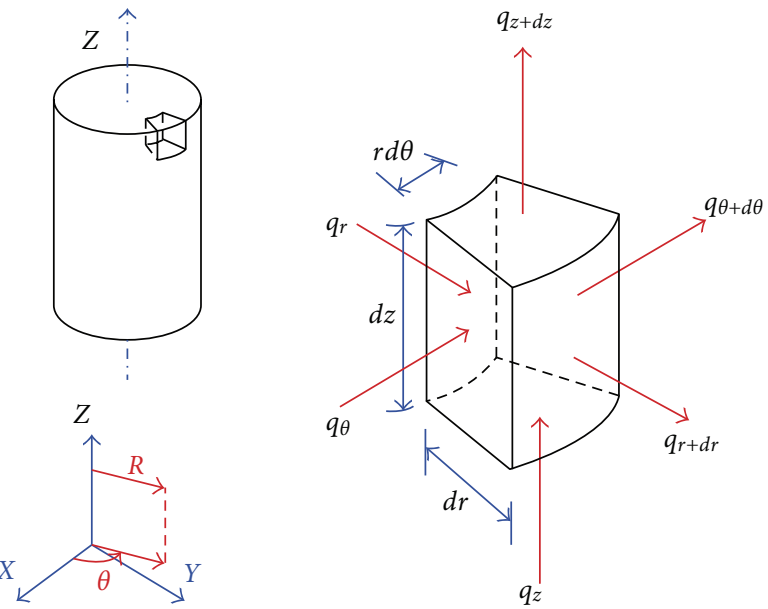

FIgURe 6: Axisymmetric element from an axisymmetric body.

The natural coordinates $L_{1}$ and $L_{2}$ are also the shape functions for the line element, thus:

$$
\begin{gathered}
S_{i}=\left(\frac{R_{j}-R}{R_{j}-R_{i}}\right)=\left(\frac{R_{j}-R}{L}\right)=L_{1}, \\
S_{j}=\left(\frac{R-R_{i}}{R_{j}-R_{i}}\right)=\left(\frac{R-R_{i}}{L}\right)=L_{2}, \\
S_{i}=L_{1}, \quad S_{j}=L_{2}, \\
R=R_{j} L_{2}+R_{i} L_{1}=R_{i} S_{i}+R_{j} S_{j}, \\
\frac{\partial[S]_{i}}{\partial r}=\frac{\partial L_{1}}{\partial r}=\frac{-1}{R_{j}-R_{i}}=-\frac{1}{L}, \\
\frac{\partial[S]_{j}}{\partial r}=\frac{\partial L_{2}}{\partial r}=\frac{1}{R_{j}-R_{i}}=\frac{1}{L} .
\end{gathered}
$$

2.3. Develop Equation for All Elements of the Domain. Derivation of equation of heat transfer in axisymmetric one-dimensional line (radius) elements by pplying the conservation of energy to a differential volume cylindrical segment as shown in Figure 6,

$$
E_{\text {in }}-E_{\text {out }}+E_{\text {generated }}=E_{\text {stored }} .
$$

The transient heat transfer within the component during quenching can mathematically be described by simplifying the differential volume term; the heat conduction equation is derived and given by

$\frac{1}{r} \frac{d}{d r}\left(K_{r} r \frac{d T}{d r}\right)+\frac{1}{r^{2}} \frac{d}{d \theta}\left(K_{\theta} \frac{d T}{d \theta}\right)+\frac{d}{d z}\left(K_{z} \frac{d T}{d z}\right)+q=\rho c \frac{d T}{d t}$,

$k_{r}=$ heat conductivity coefficient in $r$-direction, $\mathrm{W} / \mathrm{m} \cdot{ }^{\circ} \mathrm{C}$, $k_{\theta}=$ heat conductivity coefficient in $\theta$-direction, $\mathrm{W} / \mathrm{m} \cdot{ }^{\circ} \mathrm{C}$, $k_{z}=$ heat conductivity coefficient in $z$-direction, $\mathrm{W} / \mathrm{m} \cdot{ }^{\circ} \mathrm{C}$, $T=$ temperature, ${ }^{\circ} \mathrm{C}, q=$ heat generation, $\mathrm{W} / \mathrm{m}^{3}, \rho=$ mass density, $\mathrm{kg} / \mathrm{m}^{3}, c=$ specific heat of the medium, $\mathrm{J} / \mathrm{kg} \cdot \mathrm{K}, t=$ time, s. 
The assumptions made in this problem were:

(i) for axisymmetric situations one dimensional line (radius) element, there is no variation of temperature in the $\mathbf{Z}$-direction as shown in Figures 1, 2, and 3. Because we already assumed that in steel quenching and cooling process of the steel bar is insulated from convection at the cross section of the front and back.

It means that we have convection and radiation at one node only which is on the surface (node 5), in our research we focus to calculate LHP which is at (node 1), where it is the last point will be cooled, this give the maximum advantage to make our assumption more safe, because it is the last point which will effect by convection and radiation from the front and back cross-section of the steel bar therefore we can write, $(\partial T / \partial z=0)$

(ii) for axisymmetric situations, there is no variation of temperature in the $\boldsymbol{\theta}$-direction, because it is clear from Figures 1, 2, and 3 that the temperature distribution along the radius will be the same if the radius move with angle $\theta$, $360^{\circ}$ therefore, $(\partial T / \partial \theta=0)$,

(iii) the thermal energy generation rate $\dot{q}$ represent the rate of the conversion of energy from electrical, chemical, nuclear, or electromagnetic forms to thermal energy within the volume of the system. Such as conversion is the electric field which will be studied with details in the 2nd part of our research, however in this manuscript no heat generation therefore,

$$
\dot{q}=0
$$

After simplifying, (11) becomes

$$
\frac{k}{r} \frac{\partial}{\partial r}\left(r \frac{\partial T}{\partial r}\right)=\rho c \frac{\partial T}{\partial t}
$$

and also known as residual or partial differential equation

$$
\{\mathfrak{R}\}=\frac{k}{r} \frac{\partial}{\partial r}\left(r \frac{\partial T}{\partial r}\right)-\rho c \frac{\partial T}{\partial t}=0 .
$$

2.4. Galerkin Weighted Residual Method Formulation. From the derived heat conduction equation, the Galerkin residual for 1-dimensional line (radius) element in an unsteady state heat transfer by integration the shape functions times the residual which minimize the residual to zero becomes

$$
\int_{V}[S]^{T}\{\mathfrak{R}\}^{(e)} d v=0
$$

where $[S]^{T}=$ the transpose of the shape function matrix, $\{\mathfrak{R}\}^{(e)}=$ the residual contributed by element $(e)$ to the final system of equations

$$
\frac{k}{r} \int[S]^{T} \frac{\partial}{\partial r}\left(r \frac{\partial T}{\partial r}\right) d v-\int[S]^{T} \rho c \frac{\partial T}{\partial t} d v=0 .
$$

2.5. Chain Rule. The terms 1 and 2 of (16) can be rearranged using the chain rule which states that;

$$
(f g)^{-}=f g^{-}+g f^{-} .
$$

Therefore, $f g^{-}=(f g)^{-}-f^{-} g$,

$$
\frac{\partial}{\partial r}\left([S]^{T} r \frac{\partial T}{\partial r}\right)=[S]^{T}\left\{\frac{\partial}{\partial r}\left(r \frac{\partial T}{\partial r}\right)\right\}+r \frac{\partial T}{\partial r} \frac{\partial[S]^{T}}{\partial r} .
$$

Term 1 of (16) is rearranged, thus

$$
[S]^{T}\left\{\frac{\partial}{\partial r}\left(r \frac{\partial T}{\partial r}\right)\right\}=\frac{\partial}{\partial r}\left([S]^{T} r \frac{\partial T}{\partial r}\right)-r \frac{\partial T}{\partial r} \frac{\partial[S]^{T}}{\partial r} .
$$

By substituting (19) in to (16), get

$$
\begin{gathered}
=\overbrace{\frac{k}{r} \int\left\{\frac{\partial}{\partial r}\left([S]^{T} r \frac{\partial T}{\partial r}\right)\right\} d v}^{A}-\overbrace{\frac{k}{r}\left\{\left\{\frac{\partial[S]^{T}}{\partial r} r \frac{\partial T}{\partial r}\right\} d v\right.}^{B} \\
-\underbrace{\int[S]^{T}\left\{\rho c \frac{\partial T}{\partial t}\right\} d v}_{C} .
\end{gathered}
$$

Term $A$ is the heat convection terms and contributes to the conductance and thermal load matrix. Term $B$ is the heat conduction terms and contributes to the conductance matrix. Term $C$ is the transient equation and contributes to the capacitance matrix, where

$$
\begin{aligned}
\overbrace{\frac{k}{r} \int \frac{\partial}{\partial r}\left([S]^{T} r \frac{\partial T}{\partial r}\right) d V}^{A} \\
=\underbrace{-2 \pi h r z[S]^{T}[S]\{T\}^{e}}_{1}+\underbrace{2 \pi h r z[S]^{T} T_{f}}_{2} \\
\quad-\underbrace{2 \pi r z \mathcal{E}_{s} \sigma[S]^{T}[S]\{T\}^{e}\left([S]\{T\}^{e}\right)^{3}}_{3}+\underbrace{2 \pi r z \mathcal{E}_{s} \sigma[S]^{T} T_{f}^{4}}_{4} .
\end{aligned}
$$

Note that term (1) and term (3) contributed to the conductance matrix since they contains the unknown temperature $\{T\}$. Terms (2) and (4) contributed to the thermal load matrix as $T_{f}$ is the known fluid temperature. Term (3) and term (4) heat radiation are very important if our heat treatment is annealing (cooling in the furnace) or normalizing (cooling in air or jet air), but can be ignore (neglect) if the cooling is quenching in water as in our paper.

From earlier explanations, derivation and after simplifying we can formulate the conductance matrix in the $r$ direction for Term $B$ finally we get:

Term $B$ (the conduction term) contributes to the conductance matrix:

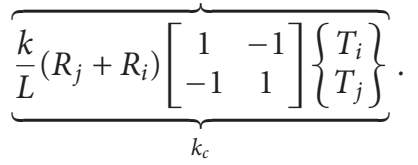

Similarly, Term $C$ is the unsteady state (transient) which contributes to the Capacitance Matrix, 
Term $C$ (heat stored) contributes to the Capacitance Matrix:

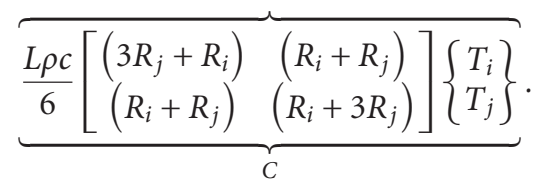

Term A (heat convection):

(i) Term $A_{1}$ : contributes to conductance matrix,

Term $A_{1}$ (the convection term) contributes to the conductance matrix:

$$
\underbrace{-2 h R_{j}\left[\begin{array}{ll}
0 & 0 \\
0 & 1
\end{array}\right]\left\{\begin{array}{c}
T_{i} \\
T_{j}
\end{array}\right\}}_{k_{h}},
$$

(ii) Term $A_{2}$ : contributes to thermal load Matrix:

Term $A_{2}$ (the convection term) contributes to thermal load matrix

$$
\underbrace{\overbrace{-2 h R_{j} T_{w}\left[\begin{array}{l}
0 \\
1
\end{array}\right]}}_{f_{h}} .
$$

2.6. Construct the Element Matrices to the Global Matrix. Assemble the global, conductance, capacitance, and thermal load matrices and the global of the unknown temperature matrix for all the elements in the domain, that is, the element's conductance, capacitance and thermal load matrices have been derived. Assembling these elements is necessary in all finite element analysis.

Constructing these elements will result into the following finite element equation:

$$
[\mathbf{K}]^{(\mathbf{G})}\{\mathbf{T}\}^{(\mathbf{G})}+[\mathbf{C}]^{(\mathbf{G})}\{\dot{\mathbf{T}}\}^{(\mathbf{G})}=\{\mathbf{F}\}^{(\mathbf{G})},
$$

with $[\mathbf{K}]=\left[\mathbf{K}_{\mathbf{c}}\right]+\left[\mathbf{K}_{\mathbf{h}}\right]$. Conductance matrix due to conduction (elements 1 to 4 ) and heat loss through convection at the element's boundary (element 4 node 5) as shown in Figures 1 and 2 :

$\{T\}:$ Temperature value at each node, ${ }^{\circ} \mathrm{C}$,

$[C]$ : Capacitance matrix, due to transient equation (heat stored),

$\{\dot{T}\}$ : Temperature rate for each node, ${ }^{\circ} \mathrm{C} / \mathrm{s}$,

$\{F\}=\left\{F_{h}\right\}+\left\{F_{\dot{q}}\right\}$ : heat load due to heat loss through convection at the element's boundary (element 4 node 5 ) and internal heat generation (element 4 node $5)$.
2.7. Euler's Method. Two point recurrence formulas will allow us to compute the nodal temperatures as a function of time. In this paper, Euler's method which known as the backward difference scheme (BDS) will be used to determine the rate of change in temperature, the temperature history at any point (node) of the steel bar [3].

If the derivative of $\mathrm{T}$ with respect to time $\mathrm{t}$ is written in the backward direction and if the time step is not equal to zero $(\Delta t \neq 0)$, we have that,

$$
\{\dot{T}\} \approx\left\{\frac{T(t)-T(t-\Delta t)}{\Delta t}\right\},
$$

with $\dot{T}=$ temperature rate $\left({ }^{\circ} \mathrm{C} / \mathrm{s}\right) ; T(t)=$ temperature at $t$ $\mathrm{s}\left({ }^{\circ} \mathrm{C}\right) ; T(t-\Delta t)=$ temperature at $(t-\Delta t) \mathrm{s},\left({ }^{\circ} \mathrm{C}\right) \Delta t=$ selected time step (s) and $\mathrm{t}=$ time (s) (at starting time, $t=0$ ).

By substituting the value of $\{\dot{T}\}$ into the finite element global equation, we have that

$[K]^{(G)}\{T(t)\}^{(G)}+[C]^{(G)}\left\{\frac{T(t)-T(t-\Delta t)}{\Delta t}\right\}^{(G)}=\{F(t)\}^{(G)}$.

Finally, the matrices become

$$
\left[[K]^{(G)} \Delta t+[C]^{(G)}\right]\{T\}_{i+1}^{(G)}=[C]^{(G)}\{T\}_{i}^{(G)}+\{F\}_{i+1}^{(G)} \Delta t .
$$

From (29) all the right hand side is completely known at time $t$, including $t=0$ for which the initial condition apply.

Therefore, the nodal temperature can be obtained for a subsequent time given the temperature for the preceding time.

Once the temperature history is known the important mechanical properties of the steel bar can be obtained such as hardness and strength.

\section{Application}

3.1. Calculation the Temperature History. The present developed mathematical model is programmed using MATLAB to simulate the results of the temperature distribution with respect to time in transient state heat transfer of the industrial quenched steel bar. The cylindrical chromium steel bar has been heated to $850^{\circ} \mathrm{C}$. Then being quenched in water with $T_{\text {water }}=32^{\circ} \mathrm{C}$, the water convection heat transfer coefficient, $h_{\text {water }}=5000 \mathrm{~W} / \mathrm{m}^{2} \cdot{ }^{\circ} \mathrm{C}$.

The temperature history at any point (node) of the cylindrical steel bar after quenched is being identified in Figures 6 and 7. The cylindrical bar was made from chromium steel $8650 \mathrm{H}$, with properties as mentioned below.

Thermal capacity, $\rho \mathrm{c}\left(\mathrm{J} / \mathrm{m}^{3} \cdot{ }^{\circ} \mathrm{C}\right)$ :

$$
\begin{aligned}
& 0 \leq T \leq 650^{\circ} \mathrm{C}, \rho c=(0.004 T+3.3) \times 10^{6}, \\
& 650<T \leq 725^{\circ} \mathrm{C}, \rho c=(0.068 T-38.3) \times 10^{6}, \\
& 725<T \leq 800^{\circ} \mathrm{C}, \rho c=(-0.086 \mathrm{~T}+73.55) \times 10^{6}, \\
& T>800^{\circ} \mathrm{C}, \rho c=7.55 \times 10^{6} .
\end{aligned}
$$

Thermal conductivity, $\mathrm{k}\left(\mathrm{W} / \mathrm{m} \cdot{ }^{\circ} \mathrm{C}\right)$ 


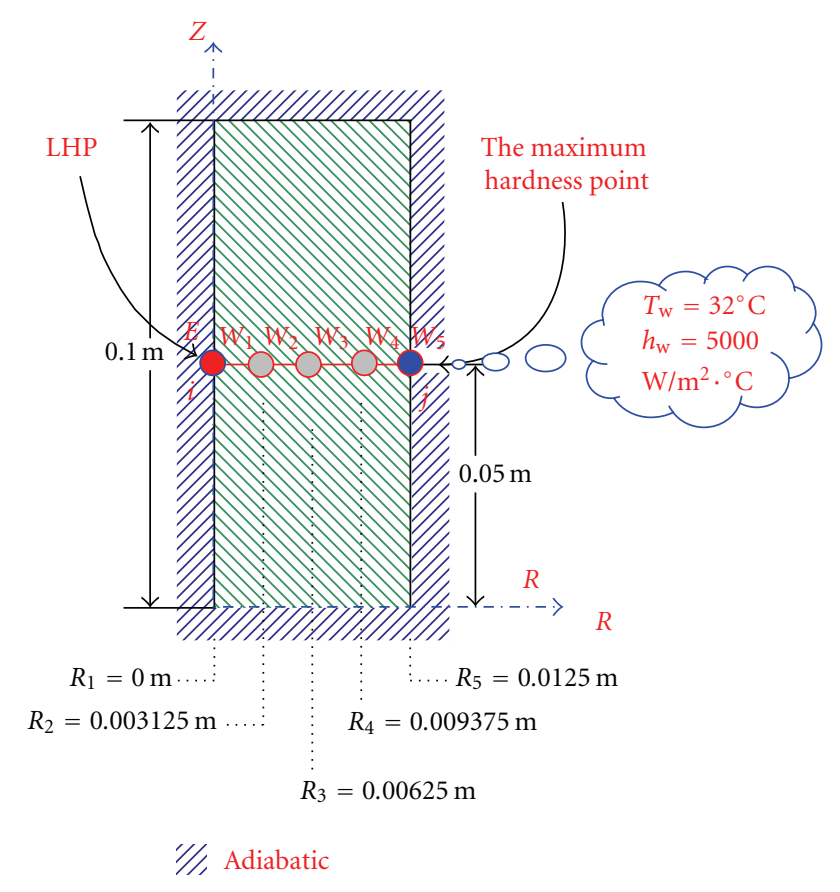

FIgURE 7: The axisymmetric one dimensional line (radius) element from the domain, the selected 4 elements with 5 nodes and the boundary at node $j(5)$ for an element 4 .

$$
\begin{aligned}
& 0 \leq T \leq 900^{\circ} \mathrm{C}, k=-0.022 T+48, \\
& T>900^{\circ} \mathrm{C}, k=28.2,
\end{aligned}
$$

where in our case the global conductance matrix $[K]^{(G)}$, the global capacitance matrix $[C]^{(G)}$ and the global thermal load matrix $\{F\}^{(G)}$ can be computed easily as the following:

$$
[K]^{(G)}=\left[K_{c}\right]^{(1)}+\left[K_{c}\right]^{(2)}+\left[K_{c}\right]^{(3)}+\left[K_{c}\right]^{(4)}+\left[K_{h}\right]^{(4)},
$$

where $\left[K_{\mathrm{c}}\right]^{(1)},\left[K_{\mathrm{c}}\right]^{(2)},\left[K_{\mathrm{c}}\right]^{(3)},\left[K_{\mathrm{c}}\right]^{(4)}$ are the conductance matrices due to conduction in 1-dimensional element for the 1 st element, the $2 \mathrm{nd}$, the $3 \mathrm{rd}$, and the 4 th element respectively while $\left[K_{h}\right]^{(4)}$ because we note that there is convection in element 4 at node $j(5)$ only as shown clearly in Figures 3 and 7:

$$
[C]^{(G)}=[C]^{(1)}+[C]^{(2)}+[C]^{(3)}+[C]^{(4)},
$$

where $[C]^{(1)},[C]^{(2)},[C]^{(3)},[C]^{(4)}$ are the capacitance matrices due to transient [unsteady state] in 1-dimensional line (radius) element:

$$
\{F\}^{(G)}=\left\{F_{h}\right\}^{(4)} .
$$

We have convection in element 4 at node $j(5)$ only as shown clearly in Figures 3 and 7.

With the input data and boundary conditions provided, a sensitivity analysis is carried out with the developed program to obtain the temperature distribution at any point (node)
TABLE 1: Cooling time and rate of cooling for each node of 1dimensional line (radius) element.

\begin{tabular}{lcc}
\hline Node & $t_{c}(\mathrm{~s})$ & $\mathrm{ROC}\left({ }^{\circ} \mathrm{C} / \mathrm{s}\right)$ \\
\hline$W_{1}$ & 8.245 & 36.38568 \\
$W_{2}$ & 8.138 & 36.864094 \\
$W_{3}$ & 7.406 & 40.507696 \\
$W_{4}$ & 5.311 & 56.486537 \\
$W_{5}$ & 1.93 & 155.44041 \\
\hline
\end{tabular}

of the quenched steel bar, as example, is the transient state temperature distribution results of the selected five nodes from the center $\left[W_{1}\right]$ to the surface $\left[W_{5}\right]$ of the quenched steed bar which were computed as shown in Figures 7 and 8.

\subsection{LHP Calculation}

3.2.1. Calculating the Cooling Time Required. In this study, we choose to calculate the cooling time between $800^{\circ} \mathrm{C}$ and $500^{\circ} \mathrm{C}[3,7-9]$, where the characteristic cooling time, relevant for phase transformation in most structural steels is the time of cooling from 800 to $500^{\circ} \mathrm{C}$ (time $t_{8 / 5}$ ) [10-17]:

$$
t_{c}=t_{800}-t_{500}
$$

From Figure 7 we can determine the time taken for node $W_{1}$ to reach $800^{\circ} \mathrm{C}$,

$$
t_{800}{ }^{\circ} \mathrm{C}=4.830 \mathrm{sec} .
$$

By the same way the time taken for node $W_{1}$ to reach $500^{\circ} \mathrm{C}$ is $t_{500^{\circ} \mathrm{C}}=13.075 \mathrm{sec}$.

So the Cooling time $t_{c}$ for node $W_{1}$;

$$
t_{c}=t_{500^{\circ} \mathrm{C}}-t_{800^{\circ} \mathrm{C}}=13.075-4.830=8.245 \mathrm{sec} .
$$

For nodes $W_{2}$ to $W_{5}$, the cooling time $t_{c}$ calculated by the same way, the final results shown in Table 1.

Table 1 shows the cooling time $t_{c}$ and the rate of cooling ROC.

\subsubsection{Calculating the Jominy Distance from Standard Jominy} Distance versus Cooling Time Curve. Cooling time, $t_{c}$, obtained will now be substituted into the Jominy distance versus cooling time curve to get the correspondent Jominy distance. Jominy distance can also be calculated by using polynomial expressions via polynomial regression via Microsoft Excel.

The standard Table (cooling rate at each Jominy distance (Chandler, 1999)) can be used too [18].

Then Jominy distance of nodes $W_{1}$ to $W_{5}$ will be calculated, the final results shown in Table 2, where the rate of cooling, ROC, was defined by;

$$
\mathrm{ROC}=\frac{800{ }^{\circ} \mathrm{C}-500{ }^{\circ} \mathrm{C}}{t_{c}}=\frac{800^{\circ} \mathrm{C}-500^{\circ} \mathrm{C}}{t_{500}{ }^{\circ} \mathrm{C}-t_{800}{ }^{\circ} \mathrm{C}}\left({ }^{\circ} \mathrm{C} / \mathrm{s}\right) .
$$

3.2.3. Predict the Hardness of the Quenched Steel Bar. The HRC of AISI-SAE $8650 \mathrm{H}$ can be calculated by using the relation between the J-Distance and the HRC from the Practical 


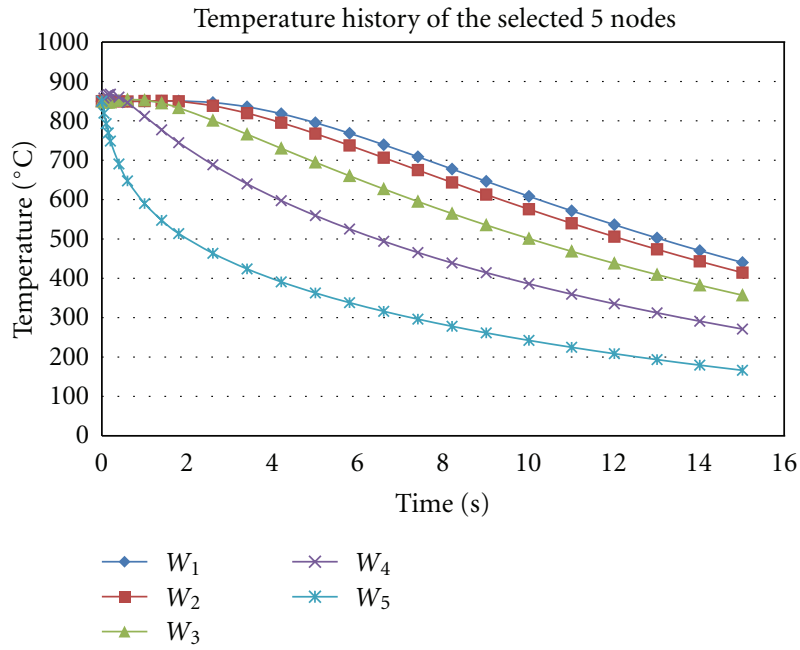

FIGURE 8: Graph of temperature history along WW cross-section from MATLAB program.

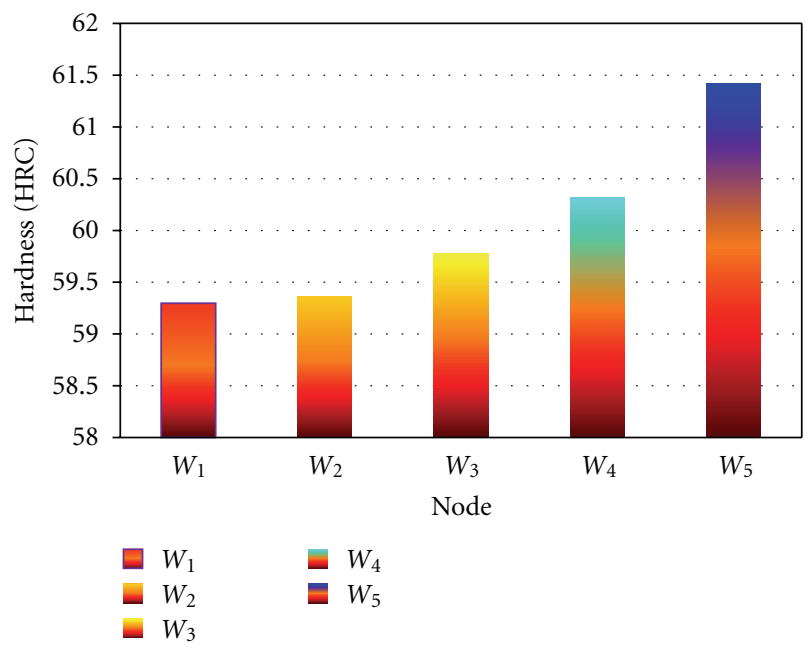

Figure 9: Hardness distribution along $W W$ cross-section for the nodes $W_{1}$ to $W_{5}$ from the centre to the surface, respectively, at half the length at the centre of the quenched steel bar.

date Handbook, the Timken Company 1835 Duebex Avenue SW Canton, Ohio 44706-2798 1-800-223, the final results are shown in Figure 9 and Table 3.

\section{Mathematical Model Verification}

The same data input for the steel properties and boundary condition used in the mathematical model is applied to the ANSYS software to verify the temperature simulation results. The temperature distribution from the ANSYS analysis is depicted figuratively as shown in Figures 10(a) and 10(b).

The temperature time graph from the ANSYS analysis is depicted as shown in Figure 11.

From the graphs shown in Figure 8 by mathematical model and Figure 11 by ANSYS, it can be clearly seen that the temperature history of the quenched steel bar have
Table 2: Cooling time, Cooling rate, and Jominy distance for the nodes $W_{1}$ to $W_{5}$.

\begin{tabular}{lccc}
\hline Node & $t_{c}(\mathrm{~s})$ & $\mathrm{ROC}\left({ }^{\circ} \mathrm{C} / \mathrm{s}\right)$ & Jominy distance $(\mathrm{mm})$ \\
\hline$W_{1}$ & 8.245 & 36.38568 & 8.685 \\
$W_{2}$ & 8.138 & 36.864094 & 8.621 \\
$W_{3}$ & 7.406 & 40.507696 & 8.179 \\
$W_{4}$ & 5.311 & 56.486537 & 6.935 \\
$W_{5}$ & 1.93 & 155.44041 & 3.431 \\
\hline
\end{tabular}

TABle 3: Cooling time, cooling rate, Jominy distance, and HRC for the nodes $W_{1}$ to $W_{5}$, water cooled.

\begin{tabular}{lcccc}
\hline Node & $t_{c}(\mathrm{~s})$ & ROC $\left({ }^{\circ} \mathrm{C} / \mathrm{s}\right)$ & $\begin{array}{c}\text { Jominy distance } \\
(\mathrm{mm})\end{array}$ & $\begin{array}{c}\text { Hardness } \\
(\mathrm{HRC})\end{array}$ \\
\hline$W_{1}$ & 8.245 & 36.38568 & 8.685 & 59.297 \\
$W_{2}$ & 8.138 & 36.864094 & 8.621 & 59.359 \\
$W_{3}$ & 7.406 & 40.507696 & 8.179 & 59.775 \\
$W_{4}$ & 5.311 & 56.486537 & 6.935 & 60.316 \\
$W_{5}$ & 1.93 & 155.44041 & 3.431 & 61.421 \\
\hline
\end{tabular}

TABLE 4: Cooling time, Cooling rate, Jominy distance, and HRC for the nodes $A_{1}$ to $A_{5}$, water cooled by ANSYS.

\begin{tabular}{lcccc}
\hline Node & Cooling time & Cooling rate & J distance $(\mathrm{mm})$ & HRC \\
\hline$A_{1}$ & 9.7 & 30.87574 & 9.556 & 58.492 \\
$A_{2}$ & 9.589 & 31.284 & 9.472 & 58.518 \\
$A_{3}$ & 9.055 & 33.130 & 9.174 & 58.613 \\
$A_{4}$ & 5.905 & 50.797 & 7.286 & 60.208 \\
$A_{5}$ & 2.180 & 137.580 & 3.828 & 61.295 \\
\hline
\end{tabular}

the same pattern. The heat transfer across the steel bar is uniform. From Figure 11 the cooling time, Jominy-distance, and consequently the hardness of the quenched chromium steel bar at any point (node), even the lowest hardness point (LHP) is determined by ANSYS too, the final results shown in Table 4 and Figure 12.

From our results we found that in the mathematical model for the 1st node with $W_{1}$ in the center, we found that HRC $=59.297$. While in ANSYS for the same node $A_{1}$, we found that $\mathrm{HRC}=58.492$.

And for the nodes on the surfaces $W_{5}$ and $A_{5}$, it was found that HRC $=61.421$ and 61.295 for the mathematical model and ANSYS, respectively. From the above, it can be seen that there is a strong agreement between both results. The difference between all the results of the mathematical model and the ANSYS simulations can be accounted due to the fact that the ANSYS software is commercial purpose, and thereby has some automated input data. But the developed mathematical model is precisely for a circular steel bar axisymmetric cross section. However, there is strong agreement between both results and thereby the result is validated, where the comparison indicated reliability of the proposed model.

Also the results showed that the node on the surface will be the 1st which completely cooled after quenching because 


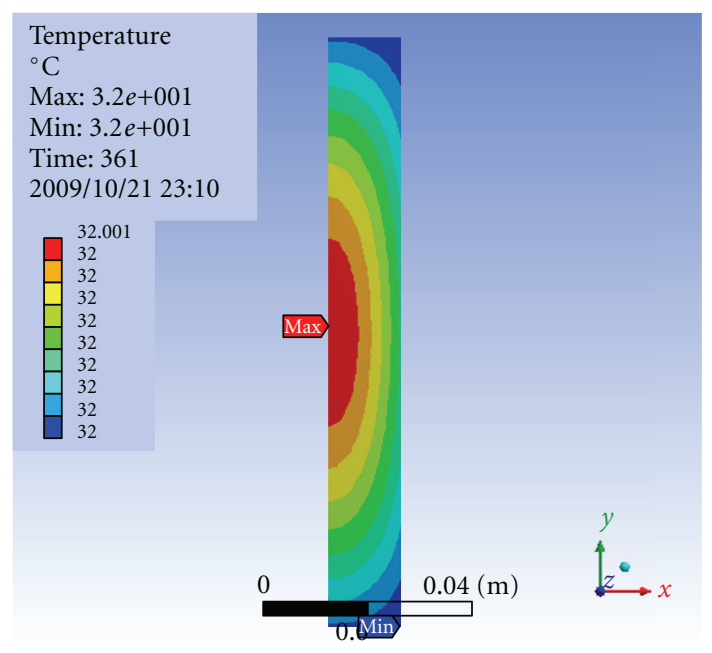

(a)

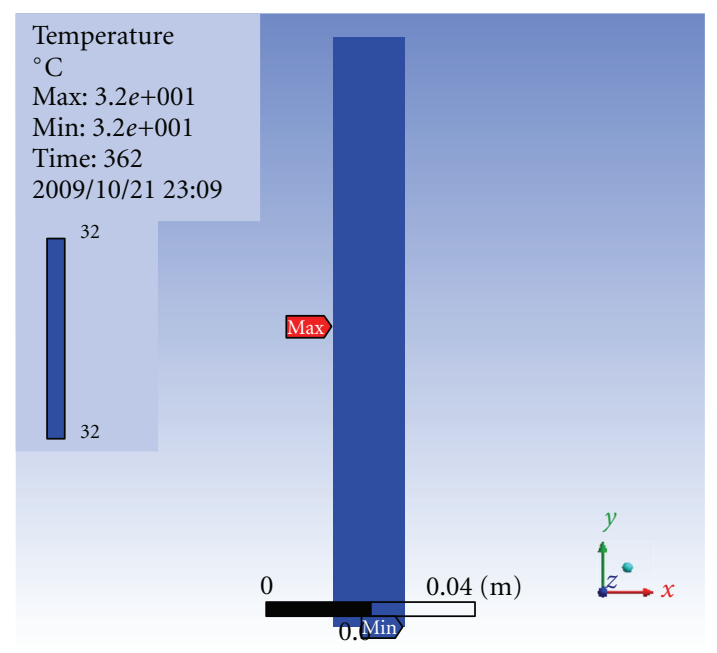

(b)

Figure 10: (a) shows the temperature distribution just before the steel bar becomes completely cooled and (b) shows the temperature distribution at the moment that the entire steel bar becomes completely cooled after $362 \mathrm{~s}$.

it is in the contact with the cooling medium then the other nodes on the radial axis to the centre, respectively, and the last point will be completely cooled after quenching will be at half the length at the centre. Hence LHP will be at half the length at the centre of the quenched industrial chromium steel bar. It will be more important to know LHP once the radius of the quenched steel bar is large because LHP will be low, in other words, it will be lower than the hardness on the surface, that means increasing the radius of the bar inversely proportional with LHP.

LHP calculation experimentally is an almost impossible task using manual calculation techniques also the earlier methods only used hardness calculated at the surface, which is higher than LHP, which has negative consequence that can result to the deformation and failure of the component.

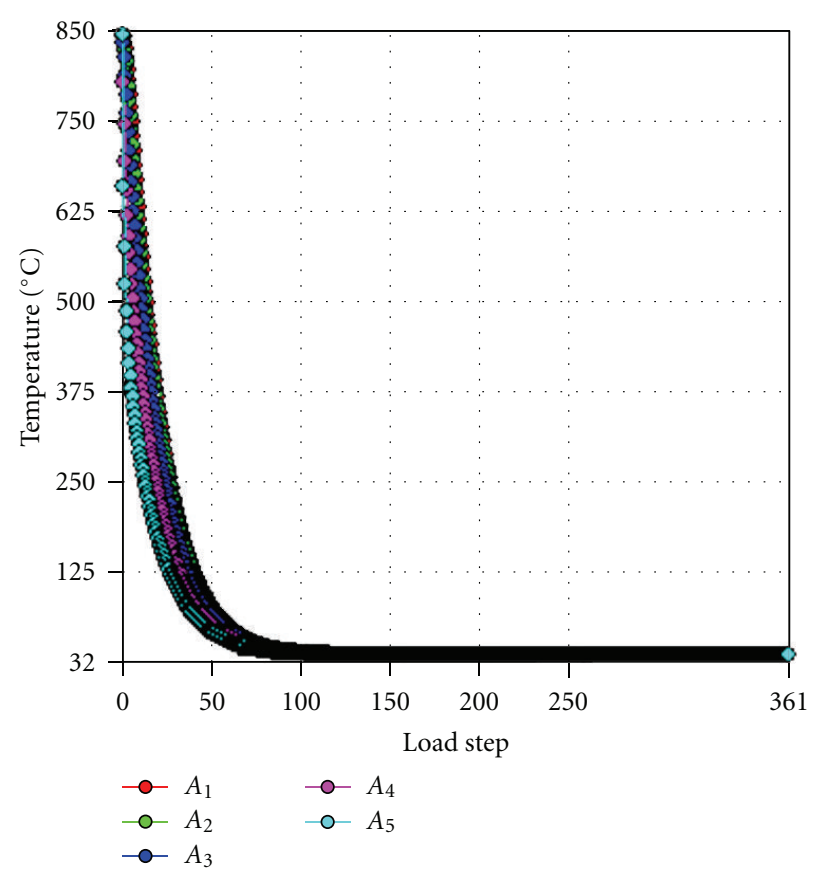

Figure 11: Temperature-time graph from ANSYS.

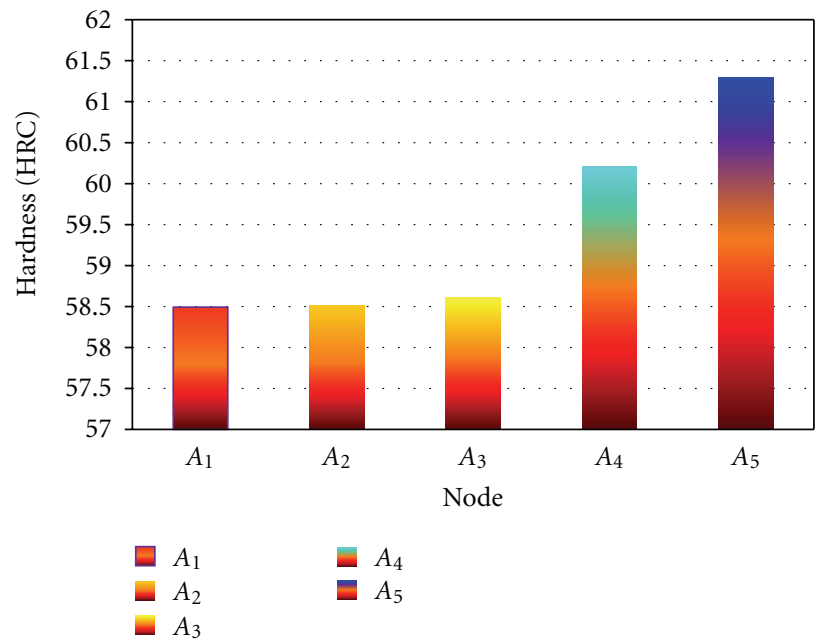

FIGURE 12: Hardness distribution by ANSYS along AA cross-section for the nodes $A_{1}$ to $A_{5}$ from the centre to the surface, respectively, at half the length at the centre of the quenched steel bar.

\section{Conclusion}

A mathematical model of steel quenching has been developed to compute LHP of the quenched chromium steel bar at any point (node) in a specimen with cylindrical geometry. The model is based on the finite element Galerkin residual method. The numerical simulation of quenching consisted of numerical simulation of temperature transient field of cooling process. This mathematical model was verified and validated by comparing the hardness results with ANSYS software simulations. From the mathematical model and ANSYS results, it is clear that the nodes on the surface $\left(W_{5}\right.$ 
and $\left.A_{5}\right)$, respectively, cools faster than the nodes on the center $\left(W_{1}\right.$ and $\left.A_{1}\right)$ because $t_{c W_{5}}<t_{c W_{1}}$ and $t_{c A_{5}}<t_{c A_{1}}$, this means that the mechanical properties will be different such as hardness where the hardness on the surface nodes $\left(W_{5}\right.$ and $\left.A_{5}\right)$ will be higher than the hardness on the center nodes $\left(W_{1}\right.$ and $\left.A_{1}\right)$.

\section{Acknowledgments}

The authors would like to thank the University Tun Hussein Onn Malaysia for supporting this research under the Science Fund Grant. The corresponding author grateful to the Postgraduate Centre of UTHM for their support of this research, where they accepted him under the university Ph.D. scholarship.

\section{References}

[1] K. N. Robert, Quenching and Tempering of Welded Steel Tubular, The Fabricator Articles, July 2001.

[2] B. Omar, M. Elshayb, and A. S. A. Elmaryami, "Unsteady state thermal Behavior of industrial quenched steel bar," in Proceedings of the 18th World IMACS Congress and International Congress on Modelling and Simulation (MODSIM '09), Cairns, Australia, 2009.

[3] E. Mohamed and Y. K. Bing, Application of Finite Difference and Finite Element Methods, University Malaysia Sabah Kota Kinabalu, Sabah, Malaysia, 2000.

[4] A. S. A. Elmaryami and B. Omar, "Developing of unsteady state axi-symmetric FEMM to predict the temperatureof industrial quenched steel," Journal of Metals Science and Heat Treatment. In press.

[5] K. G. Budinski, Engineering Material: Properties and Selection, Prentice-Hall, Upper Saddle River, NJ, USA, 4th edition, 1992.

[6] A. S. A. Elmaryami, Heat treatment of steel by developing finite element mathematical model and by simulation, M.S. thesis, University Tun Hussein Onn, Johor, Malaysia, 2010.

[7] A. S. A. Elmaryami, S. B. Haji Hasan, B. Omar, and M. Elshayeb, "Customer reviews unsteady state hardness prediction of industrial quenched steel bar [One and Two Dimensional]," in Proceedings of the Materials Science \& Technology Conference and Exhibition, Pittsburgh, Pa, USA, 2009.

[8] S. Moaveni, Finite Element Analysis; Theory and Application with ANSYS, Pearson Education International, 2008.

[9] A. Rose et al., Atlas zur Wärmebe- Handlung der Stähle I, Verlag Stahleisen, Düsseldorf, Germany, 1958.

[10] B. Smoljan, "Mathematical modelling of austenite decomposition during the quenching," in Proceedings of the 13th International Scientific Conference Achievements in Mechanical and Materials Engineering, The Polish Academy of Science, 2005.

[11] B. Smoljan, "Prediction of mechanical properties and microstructure distribution of quenched and tempered steel shaft," Journal of Materials Processing Technology, vol. 175, no. 1-3, pp. 393-397, 2006.

[12] B. Donnay, J. C. Herman, V. Leroy, U. Lotter, R. Grossterlinden, and H. Pircher, Microstructure Evolution of C-Mn Steel in the Hot Deformation Process: The Stripcam Model.

[13] B. Liščić, "System for process analysis and hardness prediction when quenching axially-symmetrical workpieces of any shape in liquid quenchants," Materials Science Forum, vol. 638-642, pp. 3966-3974, 2010.
[14] B. Smoljan, D. Iljkić, and S. Smokvina Hanza, "Computer simulation of working stress of heat treated steel specimen," Journal of Achievements in Materials and Manufacturing Engineering, vol. 34, no. 2, pp. 152-156, 2009.

[15] R. I. Hsieh, H. Y. Liou, and Y. T. Pan, "Effects of cooling time and alloying elements on the microstructure of the Gleeblesimulated heat-affected zone of $22 \% \mathrm{Cr}$ duplex stainless steels," Journal of Materials Engineering and Performance, vol. 10, no. 5, pp. 526-536, 2001.

[16] A. S. A. Elmaryami and B. Omar, "Computer simulation to predict the hardness of transient axi-symmetric industrial quenched steel bar at different radial axises," International Journal of Emerging Technology in Science and Engineering. In press.

[17] A. S. A. Elmaryami and B. Omar, "Transient computer simulation of industrial quenched steel bar to determine LHP of molybdenum and boron steel at $850^{\circ} \mathrm{C}$ as austenitizing temperature quenched in different medium," International Journal of Material Science. In press.

[18] H. Chandler, Hardness Testing Applications, Hardness Testing, ASM International, Indianapolis, Ind, USA, 2nd edition, 1999. 

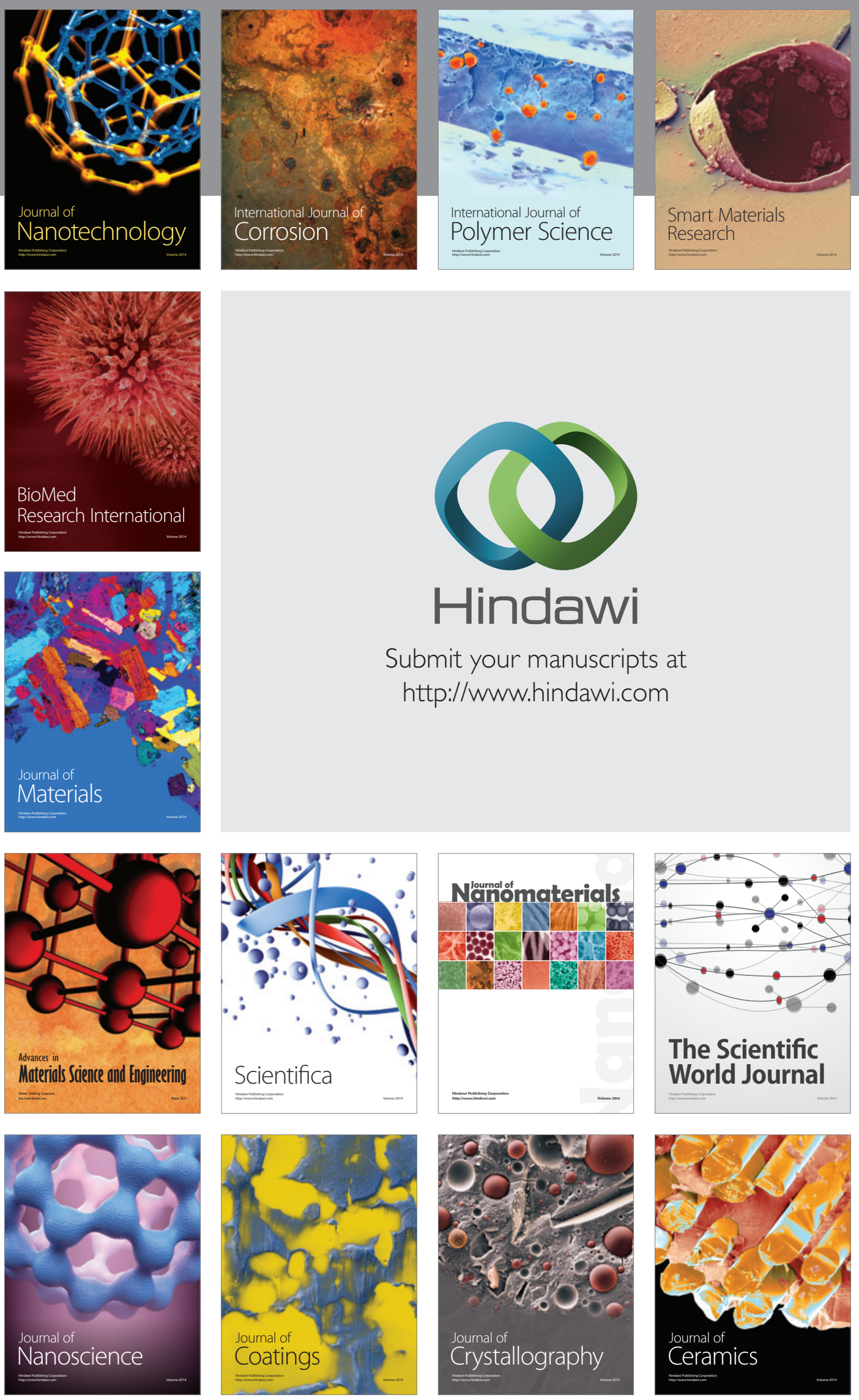

The Scientific World Journal

Submit your manuscripts at

http://www.hindawi.com

\section{World Journal}

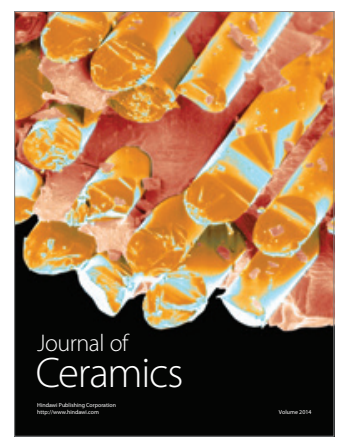

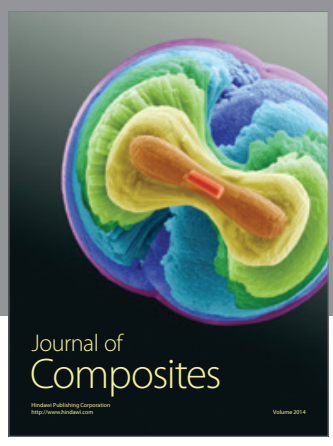
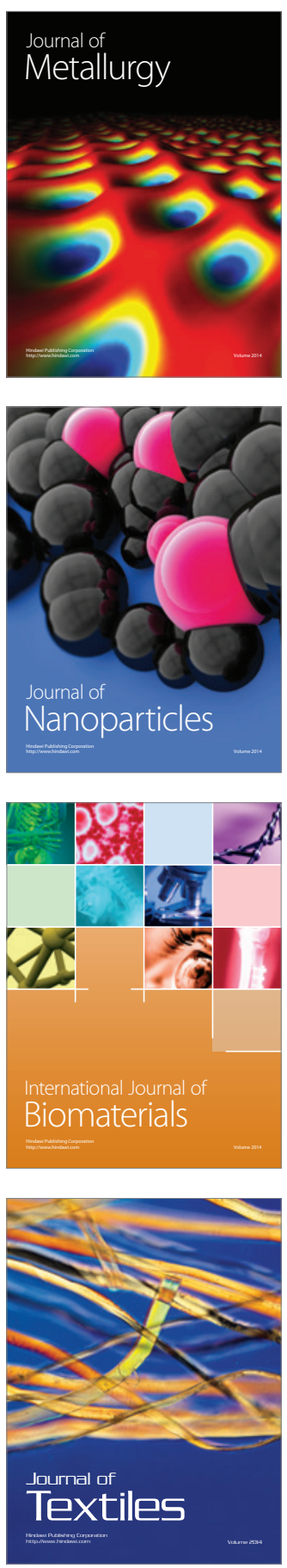